\title{
Reynolds stress models of convection in convective cores
}

\author{
Ian W Roxburgh ${ }^{1}$ and Friedrich Kupka ${ }^{2}$ \\ ${ }^{1}$ Queen Mary, University of London,UK \\ email: i.w.roxburgh@qmul.ac.uk \\ ${ }^{2}$ Max-Planck-Institute for Astrophysics, Garching, Germany \\ email: fk@mpa-garching.mpg.de
}

\begin{abstract}
We investigate the properties of non-local Reynolds stress models of turbulent convection in a spherical geometry. Regularity at the centre $r=0$ places constraints on the behaviour of $3^{r d}$ order moments. Some of the down-gradient and algebraic closure models have inconsistent behaviour at $r=0$. A combination of down-gradient and algebraic closures gives a consistent prescription that can be used to model convection in stellar cores.
\end{abstract}

Keywords. Convection, stars: interiors

\section{Reynolds Stress Models}

With no mean flow the equations for the second order correlations in a general coordinate system $\left\{x^{j}\right\}$ with metric $d s^{2}=g_{i j} d x^{i} d x^{j}$ are (cf. Canuto 1992)

$$
\begin{gathered}
\frac{\partial}{\partial t}\left(\overline{\theta^{2}}\right)+\left(\overline{u^{j} \theta^{2}}\right)_{; j}=2\left(\overline{u^{j} \theta}\right) \beta_{j}+\chi g^{j k}\left(\overline{\theta^{2}}\right)_{; j k}-2 \epsilon_{\theta} \\
\frac{\partial}{\partial t}\left(\overline{u_{i} \theta}\right)+\left(\overline{\theta u_{i} u^{j}}\right)_{; j}=\overline{u_{i} u^{j}} \beta_{j}+g_{i} \alpha \overline{\theta^{2}}-\Pi_{i}^{\theta}+\eta_{i} \\
\frac{\partial}{\partial t}\left(\overline{u_{i} u^{j}}\right)+\left(\overline{u_{i} u^{j} u^{k}}\right)_{; k}=\alpha g_{i}\left(\overline{u^{j} \theta}\right)+\alpha g^{j}\left(\overline{u_{i} \theta}\right)-\frac{2}{3} \delta_{i}^{j}\left(\overline{p u^{k}}\right)_{; k}+\nu g^{m k}\left(\overline{u_{i} u^{j}}\right)_{; m k}-\Pi_{i}^{j}-\epsilon_{i}^{j}
\end{gathered}
$$

where $\{; j\}$ denotes the covariant derivatives with respect to $x^{j}$. Here $\left(u_{1}, u_{2}, u_{3}\right)$ is the covariant velocity, $T=T_{o}+\theta$ is the temperature with $T_{o}$ the mean and $\theta$ the fluctuating component, $\nu, \chi$ the kinematic viscosity and thermometric conductivity, $\alpha=1 / T_{o}$ is the coefficient of thermal expansion, $\left(g_{1}, g_{2}, g_{3}\right)$ is the covariant acceleration due to gravity. The covariant superadiabatic temperature gradient $\left(\beta_{1}, \beta_{2}, \beta_{3}\right)$ and $\epsilon_{\theta}$ are defined as

$$
\beta_{i}=\left(\frac{\partial T_{o}}{\partial x^{i}}\right)_{\mathrm{ad}}-\frac{\partial T_{o}}{\partial x_{i}}, \quad \epsilon_{\theta}=\chi g^{j k} \overline{\theta_{; j} \theta_{; k}}
$$

For simplicity we here neglect the pressure correlations and make the approximations

$$
\eta_{i}=\frac{(\nu+\chi)}{2} g^{j k}\left(\overline{u_{i} \theta}\right)_{; j k} \quad \epsilon_{i}^{j}=\frac{2}{3} \delta_{i}^{j} \epsilon
$$

In covariant spherical polar coordinates $\left(x^{1}, x^{2}, x^{3}\right)=(r, \theta, \phi)$, the line element and covariant and contravariant velocities are

$$
\begin{gathered}
d s^{2}=g_{i j} d x^{i} d x^{j}=d r^{2}+r^{2} d \theta^{2}+r^{2} \sin ^{2} \theta d \phi^{2} \\
\left\{u_{i}\right\}=\left\{v_{r}, r v_{\theta}, r \sin \theta v_{\phi}\right\}, \quad\left\{u^{i}\right\}=\left\{v_{r}, \frac{v_{\theta}}{r}, \frac{v_{\phi}}{r \sin \theta}\right\}
\end{gathered}
$$

where $v_{r}, v_{\theta}, v_{\phi}$ are the physical components of velocity along the coordinate directions. 
With spherical symmetry the acceleration due to gravity $\left\{g_{i}\right\}=(g, 0,0)$, the superadiabatic temperature gradient $\left\{\beta_{i}\right\}=(\beta, 0,0)$, and the non vanishing correlations are

$$
\overline{\theta^{2}}, \quad \overline{v_{r}^{2}}, \quad \overline{v_{\theta}^{2}}=\overline{v_{\phi}^{2}}, \quad \overline{v_{r} \theta}, \quad \overline{v_{r}^{2} \theta}, \quad \overline{v_{\theta}^{2} \theta}=\overline{v_{\phi}^{2} \theta}, \quad \overline{v_{r} \theta^{2}}, \quad \overline{v_{r}^{3}}, \quad \overline{v_{r} v_{\theta}^{2}}=\overline{v_{r} v_{\phi}^{2}}
$$

Equations (1-3) in spherical polar coordinates then reduce to the 4 independent equations

$$
\begin{gathered}
\frac{\partial \overline{\theta^{2}}}{\partial t}+\frac{1}{r^{2}} \frac{d}{d r}\left(r^{2} \overline{v_{r} \theta^{2}}\right)=2 \beta \overline{v_{r} \theta}+\chi \frac{1}{r^{2}} \frac{d}{d r}\left(r^{2} \frac{d \overline{\theta^{2}}}{d r}\right)-2 \epsilon_{\theta} \\
\frac{\partial \overline{v_{r} \theta}}{\partial t}+\frac{d \overline{v_{r}^{2} \theta}}{d r}+2 \frac{\overline{v_{r}^{2} \theta}-\overline{v_{\theta}^{2} \theta}}{r}=\beta \overline{v_{r}^{2}}+g \alpha \overline{\theta^{2}}+\left(\frac{\nu+\chi}{2}\right) \frac{d}{d r}\left(\frac{1}{r^{2}} \frac{d}{d r}\left(r^{2} \overline{v_{r} \theta}\right)\right) \\
\frac{\partial \overline{v_{r}^{2}}}{\partial t}+\frac{d \overline{v_{r}^{3}}}{d r}+2 \frac{\overline{v_{r}^{3}}}{r}-4 \frac{\overline{v_{r} v_{\theta}^{2}}}{r}=2 \alpha g \overline{v_{r} \theta}+\nu\left[\frac{1}{r^{2}} \frac{d}{d r}\left(r^{2} \frac{d \overline{v_{r}^{2}}}{d r}\right)-4 \frac{\overline{v_{r}^{2}}-\overline{v_{\theta}^{2}}}{r^{2}}\right]-\frac{2}{3} \epsilon \\
\frac{\partial \overline{v_{\theta}^{2}}}{\partial t}+\frac{d \overline{v_{r} v_{\theta}^{2}}}{d r}+4 \frac{\overline{v_{r} v_{\theta}^{2}}}{r}=\nu\left[\frac{1}{r^{2}} \frac{d}{d r}\left(r^{2} \frac{d \overline{v_{\theta}^{2}}}{d r}\right)+2 \frac{\overline{v_{r}^{2}}-\overline{v_{\theta}^{2}}}{r^{2}}\right]-\frac{2}{3} \epsilon
\end{gathered}
$$

\section{Requirements on the 3rd order moments for regularity at $r=0$}

As $r \rightarrow 0, g, \beta \propto r$ and $\overline{\theta^{2}}, \overline{v_{r}^{2}}, \overline{v_{\theta}^{2}}$ have even expansions (with $a_{10}=a_{20}$ from (11))

$$
\overline{\theta^{2}}=a_{00}+a_{02} r^{2}+\ldots, \quad \overline{v_{r}^{2}}=a_{10}+a_{12} r^{2}+\ldots, \quad \overline{v_{\theta}^{2}}=a_{20}+a_{22} r^{2}+\ldots
$$

With regularity at $r=0$ it follows from equations (8), (9), (10), and (11) that

$$
\begin{array}{cl}
\overline{v_{r} \theta}=a_{31} r+a_{33} r^{3}+\ldots, & \overline{v_{r} \theta^{2}}=a_{41} r+a_{43} r^{3}+\ldots, \\
\overline{v_{r}^{2} \theta}=a_{52} r^{2}+a_{54} r^{4}+\ldots, & \overline{v_{\theta}^{2} \theta}=a_{62} r^{2}+a_{64} r^{4}+\ldots, \\
\overline{v_{r}^{3}}=a_{71} r+a_{73} r^{3}+\ldots, & \overline{v_{r} v_{\theta}^{2}}=a_{81} r+a_{83} r^{3}+\ldots
\end{array}
$$

\section{Down-gradient closure approximations for third order moments}

The down-gradient closures for the third order moments $\overline{u_{i} \theta^{2}}, \overline{u_{i} u_{j} \theta}, \overline{u_{i} u_{j} u_{k}}$ need to be expressed in tensorial form. For plane symmetry we take these as

$$
\overline{w \theta^{2}}=-\chi_{t} \frac{d \overline{w^{2}}}{d z}, \quad \overline{w^{2} \theta}=-\frac{\left(\nu_{t}+\chi_{t}\right)}{2} \frac{d \overline{w \theta}}{d z}, \quad \overline{w^{3}}=-\nu_{t} \frac{d \overline{w^{2}}}{d z}
$$

where $w$ is the fluctuating vertical velocity and $\nu_{t}, \chi_{t}$ are eddy transport coefficients.

In a general coordinate system with velocity $\left\{u_{i}\right\}$ these can be expressed as

$$
\begin{gathered}
\overline{u_{i} \theta^{2}}=-\chi_{t}\left(\overline{\theta^{2}}\right)_{; i}, \quad \overline{u_{i} u_{j} \theta}=-\frac{\left(\nu_{t}+\chi_{t}\right)}{2}\left(\frac{{\overline{u_{i} \theta}}_{; j}+{\overline{u_{j} \theta}}_{; i}}{2}\right) \\
\overline{u_{i} u_{j} u_{k}}=-\nu_{t}\left(\frac{{\overline{u_{i} u_{j}}}_{; k}+{\overline{u_{i} u_{k}}}_{; j}+{\overline{u_{j} u_{k}}}_{; i}}{3}\right)
\end{gathered}
$$

which are invariant under a re-ordering of indices and reduce to (16) for plane symmetry.

In spherical polar coordinates, with spherical symmetry $\left(\overline{v_{\theta}^{2}}=\overline{v_{\phi}^{2}}\right)$, these reduce to

$$
\overline{v_{r} \theta^{2}}=-\chi_{t} \frac{d}{d r}\left(\overline{\theta^{2}}\right), \quad \overline{v_{r}^{2} \theta}=-\frac{\left(\nu_{t}+\chi_{t}\right)}{2} \frac{d}{d r}\left(\overline{v_{r} \theta}\right), \quad \overline{v_{\theta}^{2} \theta}=-\frac{\left(\nu_{t}+\chi_{t}\right)}{2}\left(\frac{\overline{v_{r} \theta}}{r}\right)
$$




$$
\overline{v_{r}^{3}}=-\nu_{t} \frac{d}{d r}\left(\overline{v_{r}^{2}}\right), \quad \overline{v_{r} v_{\theta}^{2}}=-\frac{\nu_{t}}{3}\left[\frac{d}{d r}\left(\overline{v_{\theta}^{2}}\right)+\frac{2}{r}\left(\overline{v_{r}^{2}}-\overline{v_{\theta}^{2}}\right)\right]
$$

We note that the down-gradient closure approximations for $\overline{v_{r}^{2} \theta}$ and $\overline{v_{\theta}^{2} \theta}$ are incompatible with the behaviour as $r \rightarrow 0$ deduced from regularity of the governing equations, unless the flux $\overline{v_{r} \theta} \propto r^{3}$ as $r \rightarrow 0$. As both the radiative flux and the total flux $\propto r$ as $r \rightarrow 0$, this would require the core to be convectively neutral at $r=0$ (cf. Xiong, 1979).

\section{Algebraic closures for 3rd order moments}

An alternative closure procedure is to use algebraic relations as deduced by Gryanik and Hartmann (2002) which for plane symmetry are

$$
\overline{w^{2} \theta}=\frac{\overline{w^{3}} \overline{w \theta}}{\overline{w^{2}}}, \quad \overline{w \theta^{2}}=\frac{\overline{\theta^{3}} \overline{w \theta}}{\overline{\theta^{2}}}
$$

where $w$ is the fluctuating vertical velocity. In tensorial form with velocity $\left\{u_{i}\right\}$ this generalises to

$$
\overline{u_{i} u_{j} \theta}=\frac{1}{3}\left(\frac{\overline{u_{i} u_{j} u^{k}} \overline{u_{k} \theta}}{\overline{V^{2}}}+\frac{\overline{u_{i} u_{k} u^{k}} \overline{u_{j} \theta}}{\overline{V^{2}}}+\frac{\overline{u_{j} u_{k} u^{k}} \overline{u_{i} \theta}}{\overline{V^{2}}}\right), \quad \overline{u_{i} \theta^{2}}=\frac{\overline{\theta^{3}} \overline{u_{i} \theta}}{\overline{\theta^{2}}}
$$

where $\overline{V^{2}}=\overline{u_{i} u^{i}}$. For spherical symmetry this reduces to

$$
\overline{v_{r}^{2} \theta}=\frac{1}{3}\left(\frac{\overline{v_{r}^{3}}+2 \overline{v_{r} V^{2}}}{\overline{V^{2}}}\right) \overline{v_{r} \theta}, \quad \overline{v_{\theta}^{2} \theta}=\frac{1}{3} \frac{\overline{v_{r} v_{\theta}^{2}}}{\overline{V^{2}}} \overline{v_{r} \theta}, \quad \overline{v_{r} \theta^{2}}=\frac{\overline{\theta^{3}}}{\overline{\theta^{2}}} \overline{v_{r} \theta}
$$

We note that the algebraic closures give the correct behaviour $\left(\propto r^{2}\right)$ for $\overline{v_{r}^{2} \theta}, \overline{v_{\theta}^{2} \theta}$ as $r \rightarrow 0$, with the flux $\overline{v_{r} \theta} \propto r$ as $r \rightarrow 0$. However, they do not give the correct behaviour of $\overline{v_{r} \theta^{2}}$ since by symmetry $\overline{\theta^{3}} \rightarrow 0$ as $r \rightarrow 0$ so that $\overline{v_{r} \theta^{2}}$ cannot be $\propto r$. These results are independent of the form of $\overline{u_{i} u_{j} \theta}$ and the values of $a_{31}$ and $a_{33}$ in (13).

\section{A combined down-gradient and algebraic closure model}

By combining the above results we can obtain a closure approximation that at least has the correct behaviour as $r \rightarrow 0$, namely:

$$
\begin{gathered}
\overline{v_{r} \theta^{2}}=-\chi_{t} \frac{d}{d r}\left(\overline{\theta^{2}}\right), \quad \overline{v_{r}^{3}}=-\nu_{t} \frac{d}{d r}\left(\overline{v_{r}^{2}}\right), \quad \overline{v_{r} v_{\theta}^{2}}=-\frac{\nu_{t}}{3}\left[\frac{d}{d r}\left(\overline{v_{\theta}^{2}}\right)+\frac{2}{r}\left(\overline{v_{r}^{2}}-\overline{v_{\theta}^{2}}\right)\right] \\
\overline{v_{r}^{2} \theta}=\frac{1}{3}\left(\frac{\overline{v_{r}^{3}}+2 \overline{v_{r} V^{2}}}{\overline{V^{2}}}\right) \overline{v_{r} \theta}, \quad \overline{v_{\theta}^{2} \theta}=\frac{1}{3} \frac{\overline{v_{r} v_{\theta}^{2}}}{\overline{V^{2}}} \overline{v_{r} \theta}
\end{gathered}
$$

where $\overline{V^{2}}=\overline{v_{r}^{2}}+\overline{v_{\theta}^{2}}+\overline{v_{\phi}^{2}}$. This could be used in models of convection in stellar cores.

Here we have only considered closure approximations for third order moments. It would be desirable to develop the equations for third order moments in spherical geometry and to use algebraic closures on the fourth order moments. This is work in progress.

\section{References}

Canuto, V. M., 1992, ApJ 392, 218

Gryanik, V. M. \& Hartmann, J., 2002, J. Atmos. Sci. 59, 2729

Xiong, D. R., 1979, Acta. Astr. Sinica 20, 238 